\title{
Disease mongering, el lucrativo negocio de la promoción de enfermedades
}

\author{
ME. Morell Sixto ${ }^{a}$, C. Martínez González ${ }^{b}$, JL. Quintana Gómez \\ ${ }^{\circ}$ Médico de Familia. ${ }^{\circ P e d i a t r a . ~}$ \\ CS San Blas. Servicio Madrileño de Salud, Área I0. Parla, Madrid. España. \\ 'Médico de Familia. CS El Greco. Servicio Madrileño de Salud, Área 10. Getafe, Madrid. España.
}

Rev Pediatr Aten Primaria. 2009; / I:49/-5 /2

M. ${ }^{a}$ Elisa Morell Sixto, emorell.gapm I0@salud.madrid.org

\begin{abstract}
Resumen
En los países ricos vivimos una paradoja casi esquizofrénica: a pesar de tener mayor calidad y esperanza de vida con mejores indicadores de salud, la sensación de enfermedad y la dependencia del sistema sanitario es cada vez mayor. Es un hecho innegable que hemos cambiado la forma de valorar la salud y la enfermedad, diluyéndose los límites entre ambos y pasando a ser la salud un bien de consumo más.

Entre otros, la industria farmacéutica tiene un papel muy importante en la medicalización de la vida, colaborando en la transformación de algunas situaciones normales en patológicas y en la génesis de enfermedades a partir de situaciones potencialmente tratables. La Pediatría no es ajena a este fenómeno; basta observar el espectacular incremento de tratamientos farmacológicos para el déficit de atención con hiperactividad, o en sentido contrario la disminución del diagnóstico de reflujo gastroesofágico, en paralelo a la desaparición de la principal alternativa de tratamiento farmacológico. Pero hay otros agentes implicados directa e indirectamente en este fenómeno de mercantilización de las enfermedades llamado disease mongering, desde el colectivo médico sanitario hasta las instituciones. Identificar y entender el problema es básico para buscar soluciones.
\end{abstract}

Palabras clave: Industria farmacéutica, Medicalización, Promoción de enfermedades.

\section{Abstract}

We live an almost schizophrenic paradox in the rich countries: even when we enjoy the best life quality and life expectancy with the best health indicators, the feeling of disease and the dependence on the sanitary system are increasing. It is a fact that we have changed the way we value health and disease, limits between them have become diffuse, health is another consumer's good.

Inter alia, pharmaceutical industry has a very important role in the medicalisation of life, collaborating in the transformation of some normal situations into pathological ones and generating diseases from potentially treatable situations. Pediatrics is involved in this phenomenon

Los autores declaran no presentar conflictos de intereses en relación con la preparación y publicación de este artículo. 
too, so you can observe how pharmacological treatments for the attention deficit and hyperactivity disorder have increased, and on the contrary, diagnosis of gastroesophageal reflux has diminished parallel to the disappearance of its main pharmacological treatment.

Nevertheless, there are other agents directly or indirectly implicated in this phenomenon of commercialization called "disease mongering", ranging from sanitary staff to institutions. It is compulsory to identify and understand the problem in order to seek solutions.

Key words: Disease mongering, Drug industry, Medicalisation.

\section{Introducción}

"Es menester no ignorar que los medicamentos tomados en brebajes no siempre resultan útiles a los enfermos, y que habitualmente perjudican a las personas sanas" (Aurelio Cornelio Celso. De Re Medica, Libro II, siglo I a.C.)

En la época actual, y en los países ricos, donde la esperanza de vida se ha prolongado espectacularmente, muchas enfermedades infecciosas han sido eliminadas gracias a las vacunas, las anomalías genéticas son detectadas antes de nacer, podemos trasplantar órganos, aliviar el dolor, reconstruir el cuerpo y muchas más cosas inimaginables hace escasas décadas. Es llamativo observar cómo la dependencia del sistema sanitario y de los agentes de salud en general es cada vez mayor.

Vivimos un cambio en la sociedad que ha modificado sus creencias y valores y en consecuencia también sus formas de considerar la enfermedad y de enfrentarse a los avatares normales de la vida. La salud, tanto en su concepto como en su vivencia, no ha escapado a estos cambios, siendo en parte un bien de consumo más, y, por tanto, sometida a criterios sociales y modas, a fuerzas políticas y económicas, incluso a las leyes de mercado, que llegan a dirigir algunas actuaciones del sistema sanitario.

En consecuencia, las expectativas y demandas de la población en relación a su salud tienen distinto enfoque tanto en el proceso de atención, como en la búsqueda de soluciones que se pretenden inmediatas frente a síntomas banales, como si la Medicina fuera una ciencia "exacta" y omnipotente. Inmersos en esta progresiva medicalización, es frecuente considerar como enfermedad pequeños problemas como la calvicie, la sudoración, la timidez, los niños movidos, etc., y casi patológicos algunos períodos evolutivos normales como el embarazo, la lactancia, la adolescencia, la menopausia, etc. Asimismo, hay un afán desmesurado en la prevención. Se ha llevado al extremo el lema "es mejor prevenir que curar", desencadenando 
una cascada de demandas de actividades preventivas que no siempre tienen evidencia de efectividad. Todo ello genera una angustia desmedida ante síntomas banales, a la vez que una percepción cada vez mayor de vulnerabilidad ante la enfermedad'.

Por otro lado, el acceso tan generalizado a Internet facilita al usuario general, con escasa capacidad crítica, mucha más información que en épocas anteriores; información que no es neutra y, en ocasiones, está manipulada por grupos de presión, generando más bien desinformación y confusión para discriminar y discernir la información veraz y de calidad.

Sobre este tejido social, nace el concepto disease mongering (DM), término anglosajón difícil de traducir, que engloba aspectos como venta, invención y fabricación de enfermedades. La traducción que más se ajusta podría ser la de "mercantilización de las enfermedades", refiriéndose a la idea de obtener beneficios económicos fomentando la conciencia de enfermedad y la necesidad de medicinas para curarse. Existen muchas vías para transformar un problema en enfermedad, y convencer a la población general de que sus síntomas menores necesitan de un tratamiento farmacológico para "curarse" ${ }^{2}$. En defi- nitiva, se trata de dónde colocar los límites para considerar y tratar como enfermedad una condición o problema normal de la vida, que en algunos extremos puede llegar a serlo; como la dismenorrea (síndrome premenstrual y trastorno disfórico premenstrual), la timidez (fobia social), la calvicie (alopecia androgénica), la infelicidad (síndrome depresivo), etc., sin perjuicio de que haya personas que sufren enfermedades reales o formas graves de esos problemas, que son los que verdaderamente pueden beneficiarse de tratamientos farmacológicos.

El término medicalización surge en los años setenta a raíz del trabajo "Némesis médica" de Ivan Illyich" ${ }^{3,4}$, que analiza los cambios producidos en la definición y los límites de algunas enfermedades para aumentar la demanda de servicios médicos, productos y drogas sanitarias. Posteriormente, en los noventa, la periodista especializada en temas médicos Lynn Palmer acuña el término disease mongering ${ }^{5}$, describiendo cómo funciona la invención de enfermedades mediante la dilución de los límites de la normalidad hasta situaciones extremas, que las transforman en enfermedades susceptibles de ser tratadas $y$, consecuentemente, de incrementar el mercado de medicamentos: "tratar de con- 
vencer a gente sana de que está enferma y a gente levemente enferma de que está muy enferma es un gran negocio" (sic).

Podemos intuir algunas consecuencias de la medicalización inapropiada, como etiquetar enfermos siendo sanos, con la correspondiente carga física y emocional que conlleva un aumento evitable de efectos secundarios de la medicación utilizada y un mayor gasto económico. Por otro lado, dedicar fondos a una determinada intervención hace que no se invierta en otra medida que pudiera ser más coste-efectiva (coste-oportunidad de la medida).

\section{Protagonistas principales}

Las compañías farmacéuticas no son los únicos actores en este "teatro". En el marketing de enfermedades intervienen distintos actores que contribuyen con distinto peso, pero que en conjunto multiplican los efectos. Hay alianzas informales entre la industria farmacéutica con grupos de médicos, líderes de opinión y grupos de pacientes, que utilizando los medios de comunicación, generan conciencia social de enfermedad acerca de un problema $y$, por tanto, necesidad de tratamientos $^{2}$.

\section{Industria farmacéutica}

La industria farmacéutica es inmensamente poderosa, una de las empresas con más beneficios del mundo y un poder fáctico frente a la clase política. El Financial Times (2002), entre otros, la valora como una de las cinco industrias más lucrativas del mundo junto con las financieras, las dedicadas a la tecnología informativa, la industria del software y las aseguradoras. Según algunos medios son incluso más rentables que el sector financiero; no es de extrañar, por tanto, que su gran poder lo ejerza en los grandes bloques económicos de EE. UU. y la Unión Europea (UE)

La industria tiene un papel estelar en esponsorizar la definición de enfermedades, promocionándolas mediante campañas de concienciación a través de los medios de comunicación, que generan en los pacientes la necesidad de buscar un tratamiento que solucione su problema. En ocasiones, financian también organizaciones de ayuda a pacientes y a grupos de consumidores, utilizando sus departamentos de relaciones públicas y marketing. Algunas de estas organizaciones financiadas actúan independientemente y sin ánimo de lucro, pero otras contribuyen al mercantilismo organizado por la compañía farmacéutica ${ }^{7,8}$. 
La industria farmacéutica influencia la prescripción de los médicos de forma directa mediante los clásicos obsequios, pago de viajes a jornadas de formación y similares, e indirecta, a través de programas de educación, financiando publicaciones científicas o grupos de investigación de instituciones académicas y sociedades científicas, los cuales serán elementos muy valiosos para la promoción de sus productos. Con frecuencia, utiliza líderes de opinión de la profesión médica por medio de cursos impartidos y artículos publicados en revistas especializadas importantes ${ }^{4,9}$. Marcia Angell, editora jefe del The New England Journal of Medicine durante más de veinte años, afirma y demuestra entre otros temas en su libro La verdad sobre las compañías farmacéuticas cómo influyen los gastos en regalos dedicados a los médicos, en los precios de los medicamentos ${ }^{10}$.

Gran parte de los estudios de investigación de fármacos tanto en universidades como en otros organismos públicos o privados están financiados por las compañías farmacéuticas que crean dichos fármacos. Aunque el hecho no significa siempre que el estudio esté sesgado, la probabilidad de que las conclusiones sean favorables al fármaco estudiado es entre cuatro y cinco veces mayor que con financiadores sin ánimo de lucro ${ }^{11}$.

\section{Medios de comunicación}

Los medios de comunicación son la mayor fuente de información de salud de la población general ${ }^{12}$, informando de nuevos avances técnicos y nuevos tratamientos. Las historias de temas relacionados con la salud son parte importante de los guiones para acaparar audiencia, y los periódicos y televisiones tienen espacios exclusivos y periodistas especializados en salud. Influyen en crear conciencia de enfermedad y fomentar el uso de los servicios sanitarios en la población, ampliando los límites de la enfermedad, aceptando de forma no crítica las definiciones de estas, sobreestimando prevalencias, subrayando titulares con casos anecdóticos sin distinguir grado de enfermedad, sugiriendo en ocasiones desconocimiento de los médicos en relación a ciertas enfermedades, o en base a listas de síntomas que inducen a los pacientes a consultar a sus médicos.

Respecto a los tratamientos, es habitual magnificar los beneficios de un fármaco en términos de "curaciones milagrosas" o usar descripciones cualitativas como "mejoría significativa". Es frecuente también minimizar los efectos secundarios o sugerir que los tratamientos a largo plazo son seguros y efectivos, ignorando la duración de los 
ensayos clínicos en los que están basados dichos tratamientos ${ }^{13}$.

En EE. UU. y Nueva Zelanda se permiten anuncios en los medios de comunicación directos al consumidor. En nuestro país no está permitida la publicidad directa (Directiva Europea 2001/83/CEE, actualizada en 2004), pero está empezando a haber una publicidad de fármacos encubierta, utilizando los mismos logos de las cajas de los fármacos o dándose pautas que indican claramente a qué fármacos se refieren; para estos anuncios, es frecuente contratar a actores de reconocido prestigio, para aportar sensación de veracidad.

Recientemente se ha publicado, y es anunciado a bombo y platillo, un estudio patrocinado por la Universidad de Nuevo México y Pfizer, sobre la eficacia del sildenafilo en mujeres que toman inhibidores de la recaptación selectiva de serotonina (ISRS) ${ }^{14}$. El estudio tiene bastantes problemas de diseño, los resultados han sido medidos con escalas con valoraciones subjetivas y además no son clínicamente trascendentes; no obstante la publicidad alcanzada es muy importante ${ }^{15-17}$.

Para contrarrestar este panorama, hay que decir que cada vez hay más periodistas escépticos que están haciendo trabajos de investigación sobre este te- ma con el suficiente rigor, cordura y responsabilidad. De hecho, han sido periodistas como Lynn Payer y Ray Moynihan, entre otros, los primeros que alzaron la voz y denunciaron públicamente el creciente negocio de las enfermedades. En España, también empiezan a aparecer en los medios de comunicación artículos dedicados a este mercadeo ${ }^{18}$.

\section{Médicos}

La profesión médica está fuertemente implicada en el DM. La industria farmacéutica gasta cantidades ingentes de dinero en la "educación" de los médicos para favorecer sus intereses, ya que evidentemente si esta "docencia" no revirtiera en beneficios para las compañías, con bastante probabilidad dejaría de ofertarse e impartirse. Un estudio publicado en los noventa comprobó que en un mes casi el $50 \%$ de los médicos utilizaban información ofrecida por los visitadores de laboratorios farmacéuticos en la atención a sus pacientes y tan solo un $1 \%$ de los médicos nunca utilizaban dicha información sesgada en su práctica profesional (figura 1) ${ }^{19}$.

Algunos médicos, fundamentalmente del ámbito de la Atención Especializada, reciben de forma positiva la aparición de nuevas enfermedades pues propor- 
Figura 1. Porcentaje de médicos que usan la información proporcionada por la industria farmacéutica en su práctica clínica.

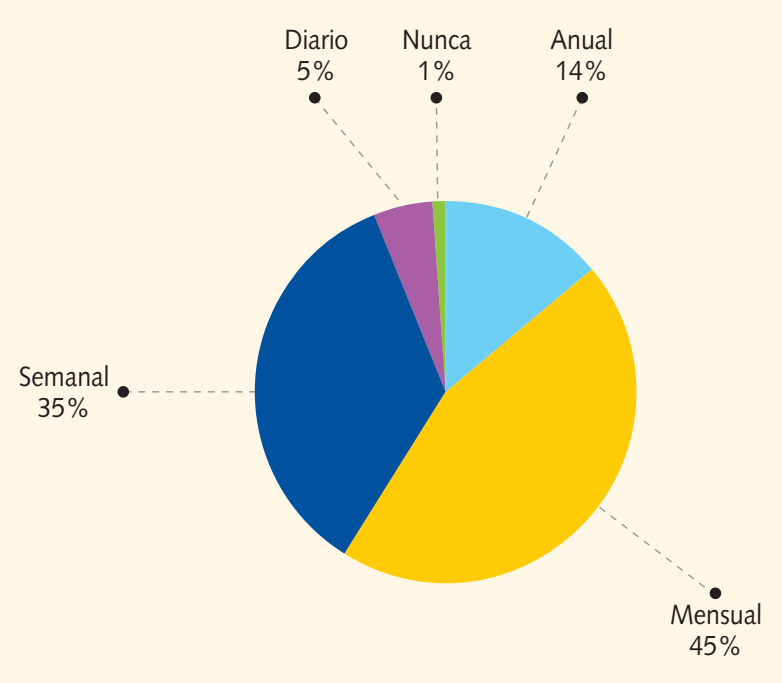

Adaptada de Caudill TS, Jonson MS, Rich EC, McKinney WP. Physicians, pharmaceutical sales representatives and the cost of prescribing. Arch Fam Med. 1996;5:201-6.

ciona estatus, influencia y nuevos horizontes de consultas monográficas con el prestigio que ello conlleva. En esta línea, los avances en genética amplían la posibilidad de "definirnos" a todos como enfermos estudiando los genes deficientes que nos predisponen a enfermedades, hecho que ha llevado a la creación de unidades de consejo genético ${ }^{20}$.

La industria farmacéutica, por su naturaleza mercantil, debe vender sus productos y utiliza sus técnicas comerciales, que pueden traspasar en no pocas oca- siones las normas éticas. Pero los médicos que se implican con ellos son igual o más responsables si se dejan manejar por este comercio. Muchos países y organizaciones han reconocido que esta no es una relación sana para la atención de los pacientes, y ya hay códigos de buena práctica en esta relación, aunque no se cumplan en bastantes ocasiones ${ }^{21}$.

A pesar de la más que demostrada evidencia de cambios en el hábito prescriptor, influenciados tanto por el discurso de los agentes comerciales como 
por las dádivas que ofrecen, todavía hay muchos médicos que participan en ello creyéndose inmunes a la seducción de la industria farmacéutica e independientes a la hora de prescribir.

\section{Políticos y administración sanitaria}

Aunque lo deseable sería que nunca se hiciera política con la sanidad y la salud, este apartado "vende" en todos los programas de grupos políticos, por lo que la protección de la salud de los ciudadanos y la oferta de prestaciones y soluciones, a veces con poca evidencia, permite que les lleguen más votos, aun a costa en ocasiones de aumentar la conciencia de enfermedad en la población.

Se permiten campañas de imagen donde se ofrecen soluciones farmacológicas financiadas a problemas que no son tales (caída de pelo y finasteride). Por otro lado, se instauran algunos programas preventivos poblacionales que carecen de evidencia científica o se instauran actividades sin suficientes datos de eficacia y seguridad.

De alguna manera los organismos gubernamentales encargados de ejercer la función de control del mercado de fármacos en beneficio de los pacientes, entran en este juego. En los últimos años, las agencias de medicamentos de los países europeos han aceptado más rápido de lo deseable la aprobación de nuevos productos a instancias de la industria farmacéutica. Es más que probable que una de las causas sea la creciente dependencia financiera de las agencias de estos clientes, por ejemplo, y según algunas fuentes, la Foods and Drugs Administration (FDA) de EE. UU. está financiada en un $75 \%$ y la Agencia Europea de Evaluación de Medicamentos en un $80 \%$ (dependiendo esta última de la Dirección General de Industria, en lugar de la de Salud) ${ }^{22}$. Así se dio el caso de retirada del mercado de la única presentación de hidroclorotiazida a dosis bajas a petición del fabricante, a pesar de que los ensayos clíni$\cos$ y metaanálisis demuestran que, hasta la fecha, los diuréticos a dosis bajas son el tratamiento de elección en el tratamiento inicial de HTA. Es verdad que a los pocos meses fue nuevamente repuesta la presentación, pero ya se había producido un importante cambio en el tratamiento de los pacientes que lo usaban ${ }^{23}$.

\section{Pacientes}

Tal como hemos señalado, hay una convicción generalizada en la población actual de que la medicina moderna y tecnológica todo lo puede, que unido, 
entre otros factores, al nivel educativo, el acceso a la información sanitaria vía Internet y a la cultura del consumismo, hace que se busquen e incluso se exijan soluciones médicas ante problemas que no lo son. Esta forma de ver la salud genera una base propicia para el mercadeo de enfermedades. En no pocas ocasiones, hay usuarios que a pesar de conocer las escasas alternativas médicas a un problema serio de salud, incluso con balance riesgo/beneficio desfavorable, insisten en usarlas "porque está incluido" o porque se niegan a aceptar la realidad. Es la llamada "regla del rescate" ${ }^{23}$ que puede llevarse a extremos, como sería el caso de actuaciones medicas éticamente no razonables en pacientes agonizantes.

Se forman grupos y asociaciones de pacientes, en principio para difundir información y defender derechos ante la sociedad y la Administración. No dudamos de las buenas intenciones y actitudes de muchos de estos grupos, pero en ocasiones, algunos ganan poder y beneficios, tanto morales como económicos, si la condición común que les agrupa es considerada y definida como enfermedad'. En no pocas ocasiones estas asociaciones están fundadas por la industria farmacéutica, y cuando esto ocurre, sus pretensiones y objetivos cambian, por no decir que el problema acerca del que están agrupados ya nunca más se cuestiona como enfermedad, y se mantiene un discurso no crítico sobre el tratamiento farmacológico disponible y sobre cómo la "enfermedad" está infradiagnosticada e infratratada. Se intenta, por tanto, maximizar la búsqueda de casos para ampliar la prevalencia de dicha condición y poder implantar un tratamiento. En el ámbito de la prevención y al respecto de la financiación por la industria farmacéutica de asociaciones, un ejemplo sería la actual promoción y venta en EE. UU. de un collar que llaman "amuleto de vida" entre adolescentes, cuyos beneficios irían a parar a la prevención del cáncer de la Prevention Cancer Foundation cuyo patrocinador principal es Sanofi-Aventis, creador de la vacuna tetravalente frente al virus del papiloma humano (VPH), Gardasil ${ }^{\otimes 24}$.

\section{Ejemplos de "fabricación" de enfermedades}

Aunque siempre hay una minoría de pacientes reales que se pueden beneficiar de los tratamientos, para "crear la necesidad" y generar esa mayoría a la que se dirigen las estrategias, existen algunas tácticas; las principales descritas por diversos autores serían ${ }^{5,25}$. 
1. Tomar una función normal y dar a entender que algo va mal en ella y que debe tratarse.

2. Atribuir un padecimiento a una enfermedad donde no la hay.

3. Aumentar los rangos de prevalencia de las enfermedades.

4. Definir una condición de salud como "enfermedad por deficiencia" o por "disbalance hormonal".

5. Fomentar el miedo en personas sanas acerca de una probable futura enfermedad.

6. Introducir nuevos diagnósticos, cuanto menos cuestionables mejor, que sean difíciles de distinguir de la vida normal.

7. Utilizar estadísticas maquillando resultados de estudios para exagerar los beneficios de tratamientos.

8. Promocionar drogas agresivas para síntomas y enfermedades leves.

9. Promocionar fármacos como soluciones de primera línea para problemas que antes no eran considerados como problemas médicos.

10. Redefinición de enfermedades usando resultados intermedios como resultados finales; por ejemplo, considerar la osteoporosis como una enfermedad en base solo a la osteopenia (resultado intermedio), o valorar la hipercolesterolemia como una enfermedad suscepti- ble de tratamiento, y no como un factor de riesgo cardiovascular.

11. Promoción de tecnologías aparentemente sin riesgos y mágicas.

\section{Convertir variantes de la normalidad en problemas médicos}

La calvicie es un ejemplo claro de cómo se han medicalizado procesos normales de la vida. De forma paralela a la aparición de la nueva presentación del fármaco finasteride de Merck (Propecia ${ }^{\oplus}$ ) para el tratamiento de la caída del pelo, comienza una campaña mediática donde se habla de los traumas emocionales, incluso del impacto laboral que conlleva la pérdida del cabello, a la vez que salen estudios y opiniones de expertos donde se sientan las bases del tratamiento. No se menciona en los medios de comunicación, que detrás de estos estudios, expertos e institutos de estudio del cabello, está la fuerte financiación de Merck .

\section{Transformar síntomas leves en enfermedades graves}

Sería el caso, entre otros, del síndrome del colon irritable que durante bastante tiempo se ha considerado un trastorno funcional y un diagnóstico de exclusión tras descartar enfermedades orgánicas. Su consideración como enfermedad cambia a partir de la estra- 
tegia de marketing por parte de la compañía GlaxoSmithKline del fármaco alosetrón (Lotronex ${ }^{\circledast}$ ). Iniciaron un programa de educación para "crear una nueva percepción del problema en la profesión médica", utilizando líderes de opinión, guías de práctica clínica y entrega de material de promoción tanto a farmacéuticos como a enfermeros y pacientes. La campaña se paró por la retirada del fármaco después de que la FDA informara de efectos adversos graves (colitis isquémicas) y en ocasiones fatales ${ }^{26}$.

\section{Considerar características}

de la personalidad o problemas sociales como enfermedad

Tanto en adultos como en niños, la timidez extrema puede en ocasiones ser un problema, llegando a la fobia social (FS). La primera descripción diferenciada de la fobia social fue en 1966 y su reconocimiento como entidad clínica independiente aparece por primera vez en el DSM-III en 1980 y en 1992 en la CIE-10. Una revisión Cochrane proporciona pruebas de que la medicación puede ser efectiva en el tratamiento de la FS a corto plazo, sobre todo con ISRS, a la vez que reconocen la posibilidad de un sesgo de publicación ${ }^{27}$. El número de estudios publicados sobre la prevalencia de la FS en población adulta es considerable; sin embargo, se han realizado pocos trabajos en la edad pediátrica, estimándose una prevalencia en adolescentes muy variable entre el $1,1 \%$ y el $11,6 \%$ en diferentes países, en España entre 6,3\% y el 8,2\%. En general, las tasas de prevalencia de los estudios que siguen los criterios del DSM-III-R y DSM-IV son más elevadas que las del DSM- $I \|^{28}$.

Son recientes los estudios con tratamientos farmacológicos en niños con FS, algunos curiosamente con otros trastornos comórbidos, incluso más importantes que la propia fobia, como la depresión ${ }^{29}$.

A pesar de los criterios diagnósticos, determinar cuándo se supera el umbral de la timidez normal, o cuándo el miedo a hablar en público o la ansiedad de ejecución constituyen estados patológicos, máxime en niños, no parece fácil, y no debería estar influenciado por la posibilidad de una alternativa farmacológica.

\section{Factores de riesgo conceptualizados como enfermedad}

La presión arterial elevada, la hipercolesterolemia y la disminución de la masa ósea son claros ejemplos de factores de riesgo que han pasado a ser considerados como enfermedad prevalente. 
En relación a la osteoporosis, la industria farmacéutica ha conseguido extender la idea de que es un "ladrón silencioso que roba el calcio de los huesos" y que si no se está alerta puede llevarnos a una vida llena de limitaciones. Desde los aspectos conceptuales controvertidos (no solo influye la densidad mineral ósea en el riesgo de fracturas, los valores de densitometría arrojan un valor predictivo positivo muy pobre en población de bajo riesgo) hasta incorrecciones de la estadística (utilización de riesgos relativos de fracturas con los tratamientos preventivos cuando el riesgo absoluto es muy pequeño en mujeres sanas, sin otros factores de riesgo) hacen considerar que la osteoporosis debería volver a su estatus inicial y no al actual, ya extendido, de enfermedad tratable?.

En el ámbito pediátrico se han producido cambios recientes en el abordaje del tratamiento farmacológico de la hipercolesterolemia, en paralelo con la preocupación en torno a la epidemia de obesidad, fundamentalmente en EE.UU. Hasta ahora la Academia Americana de Pediatría (AAP) recomendaba tratamiento farmacológico a partir de los 10 años, ahora ha disminuido la edad a 8 años ${ }^{30}$; indicaba fundamentalmente resinas, colestiramina o colestipol, cuyo efecto reside en el in- testino, ahora se incluyen las estatinas. Aunque el efecto principal de las estatinas es a nivel hepático, algunos fármacos de esta familia también inhiben la síntesis de colesterol en otros órganos como el cerebro, donde reside el $25 \%$ del total de los depósitos del cuerpo, cumpliendo importantes funciones. No olvidemos que a los 8 años el cerebro y otros órganos todavía están en período de crecimiento y que hay estudios limitados, y solo a corto plazo, sobre seguridad y estatinas en niños. Una cosa es tratar el caso excepcional del niño con hipercolesterolemia familiar, y otra extender el tratamiento a niños con riesgo cardiovascular aumentado por estilos de vida modificables ${ }^{31}$. Esta es una puerta abierta a la industria, que es más que probable que rentabilizará.

\section{Redefinir prevalencia para ampliar la extensión del problema a más población}

Un ejemplo claro lo consiguió magistralmente Pfizer con su fármaco sildenafilo $\left(\right.$ Viagra $^{\circledR}$ ) como tratamiento de la disfunción eréctil. A partir de estudios con bastantes limitaciones metodológicas amplían la prevalencia del problema, llegando a hablar en su web de más del $50 \%$ de los hombres mayores de 40 años con algún problema de erección susceptible de tratamiento, enfatizan sus 
efectos psicológicos y posteriormente se modifican los criterios de tratamiento a la vez que se amplía el mercado utilizando a los medios de comunicación. En consecuencia, en la población ha calado la idea de que cualquier desviación de una perfecta función eréctil, es una enfermedad susceptible de tratamiento con Viagra $^{\oplus 32}$.

Un problema específico de la Pediatría es el trastorno por déficit de atención e hiperactividad (TDAH), del cual, tanto profesionales sanitarios como educadores, tenemos la impresión de ir en aumento. Las cifras de prevalencia que más se repiten en los estudios están en torno al $5 \%$ de la población escolar, pero la prevalencia real es desconocida, ya que oscila con gran variabilidad entre el $2-12 \%$ de la población general33-35. Datos objetivos estiman que en EE. UU. entre los años 2000-2003, el número de menores de 19 años en tratamiento con algún fármaco por problemas de salud mental se elevó un $20 \%$, aumentando un $183 \%$ la venta de medicación frente al TDAH, un $27 \%$ los antidepresivos y un $60 \%$ los fármacos para tratar el autismo y alteraciones de la conducta ${ }^{36}$. Aunque el TDAH es una patología reconocida (CIE, DSM-IV), y los trastornos mentales qué duda cabe que existen en los niños, no podemos dejar de pensar que la posibilidad de un tratamiento farmacológico puede disparar algunos diagnósticos, hecho que no ocurre con problemas no tratables con fármacos como la dislexia. Tampoco debemos obviar que las clasificaciones de las enfermedades mentales son clasificaciones de consenso entre profesionales escogidos, algunos con conflictos de intereses, lo cual quiere decir que la base conceptual no es etiopatogénica sino descripti$\mathrm{va}^{37}$. La realidad es que el TDAH, sea un síntoma, un síndrome o una enfermedad, es un diagnóstico en el 7,8\% de niños de EE. UU. entre 4-17 años (4,4 millones de niños), según resultados de una encuesta a los padres realizada en 2003, de los cuales el $56 \%$ estaban medicados en ese momento, unos 2,5 millones de niños ${ }^{38}$; y es la causa de que en España el consumo de metilfenidato se quintuplicara de 1992 a 2001, pasando de 0,13 a 0,63 niños tratados de cada 1.000 , estimándose un aumento en el consumo anual del $8 \%{ }^{39}$. El problema surge cuando un tema inicialmente médico rápidamente se convierte en social, en parte por la presión de la sociedad en general, que siendo hiperactiva no tolera determinados comportamientos infantiles muy activos, pero también de profesionales, asociaciones de pacientes e industria. Probablemente podrían dis- 
minuir los diagnósticos, entendiendo el TDAH desde un modelo de funcionamiento mental en donde se articularán síntomas conscientes e inconscientes, más que desde un modelo basado en la conducta observable, de tal manera que, y en parte debido a la falta de tiempo de los profesionales, no se estableciera el diagnóstico en función solo de una suma de síntomas ${ }^{40}$, a veces recogidos a través de cuestionarios globales, de "banda ancha", no recomendados para el diagnóstico, por su baja sensibilidad y especificidad (AAP) ${ }^{35}$.

Es un hecho que los congresos específicos de todo tipo cuentan con la industria como entidades patrocinadoras; es el caso del III Congreso Internacional Multidisciplinar sobre TDHA celebrado en enero de $2008^{41}$, que contaba entre otros patrocinadores con los laboratorios Janssen (Concerta ${ }^{\circledR}$ ) y Juste (Medikinet $^{\oplus}$ ). Es verdad que sin patrocinio de la industria probablemente sería difícil llevar a cabo estos encuentros, pero tampoco podemos ser ingenuos en cuanto a la rentabilidad que les supone; por ejemplo, la producción lícita de metilfenidato aumentó de 2,8 toneladas en 1990, a 15,3 toneladas en $1997^{42}$. También los profesores, que tienen un papel estelar en la detección del TDAH, en función no solo de la conducta del niño, sino de cuestionarios específicos como el Conner teacher's rating scale, son objetivo de las grandes empresas farmacéuticas. A este colectivo altamente sensibilizado, dirigen sitios web, líneas telefónicas específicas y gratuitas e información repartida por los colegios, en forma de "campañas educativas específicas" ${ }^{43}$.

Finalmente, es importante la crítica fundamentada, el debate sobre el diagnóstico y el tratamiento farmacológico del TDAH, que como tal, se convierte en enfermedad tan solo en 1972, pero probablemente solo veremos la luz con el paso del tiempo.

En sentido contrario, es llamativo observar enfermedades que casi se han extinguido al desaparecer la alternativa terapéutica farmacológica. Los protocolos de estudio del lactante asmático siguen incluyendo el reflujo gastroesofágico (RGE) como posible etiología, frente a lo cual el tratamiento farmacológico habitual era el procinético cisapride. Pero desde el año 2000 al relacionarse causalmente su efecto alargador del QT con 341 casos de arritmias que incluyeron 80 muertes en pacientes tratados con cisapride ${ }^{44}$, la FDA y la European Medicines Agency (EMEA) ${ }^{45}$, tras valorar que la mayoría de los estudios en niños mayores de 36 meses no de- 
mostraban eficacia superior al placebo, restringieron su uso a neonatos y menores de 36 meses con RGE claro, tras fallar otros tratamientos. Mientras el arsenal de tratamientos farmacológicos dirigidos al asma no ha dejado de crecer, en la práctica, no se contempla descartar de forma rutinaria el RGE en el asma. ¿Qué ha pasado?, ¿ha desaparecido el problema?, o ¿hemos cambiado el enfoque diagnóstico influidos por la existencia o no de posibilidades terapéuticas farmacológicas? Cuanto menos, es un tema que merece reflexión.

\section{¿Es posible resistir a tantos intereses?}

Actualmente en todo el mundo hay movimientos para identificar, entender y frenar este problema. Pequeños pasos pueden tener cada vez más repercusión si participan todos los implicados desde todos los niveles comentados. En este sentido ya existen algunas iniciativas en curso tal como veremos.

\section{Médicos}

Es un deber moral dar a conocer a los profesionales sanitarios este problema, para tomar conciencia de su magnitud y de lo que nos puede influir a la hora de etiquetar pacientes y prescribir. Los profesionales sanitarios debemos promover el autocuidado y la autonomía de los pacientes, evitando tratamientos médicos para procesos normales de la vida que solo causen disconfort, sintomatología banal y reacciones psicológicas normales ante situaciones de la vida que inevitablemente causan estrés. Se impone pensar en términos de prevención cuaternaria, término que alude a prevenir o atenuar los efectos del exceso de la actividad sanitaria, máxime si nos referimos a enfermedades dudosas, más bien variantes de la normalidad, que han entrado en la vorágine del diagnóstico y el tratamiento poco o nada justificado ${ }^{46}$.

Algunos médicos se han convertido en pioneros de la desmedicalización, alejándose del peligroso juego de la creación de enfermedades, pero la actitud personal es insuficiente: este estilo debería ser fomentado e impulsado desde todos los ámbitos de la enseñanza médica, para que no fuera solo una excepción o una estrategia personal.

Para combatir el DM es fundamental el distanciamiento real del profesional sanitario de la industria farmacéutica, hecho que debiera estar promovido tanto por los políticos como por las agrupaciones de pacientes ${ }^{4,47}$. Hay que ser cuidadoso a la hora de elegir programas de educación y docencia impartidos por la industria. No dudamos de que muchos serán impecables en su 
planteamiento docente, pero no hay que olvidar que la industria farmacéutica invierte más del doble en marketing de productos y formación médica que en investigación y desarrollo ${ }^{48}$.

Los profesionales sanitarios deberíamos formarnos en el análisis crítico de la bibliografía científica para la interpretación correcta de los datos que aporta la industria, reconociendo las presentaciones sesgadas que en bastantes publicaciones aparecen ("maquillaje de datos").

Es necesaria la existencia de fuentes independientes de información médica, no financiadas. En este sentido hay cada vez más instituciones y publicaciones, como los boletines farmacoterapéuticos editados por las comunidades autónomas entre otros, sin ánimo de lucro. La Organización Mundial de la Salud (OMS) publicó una Guía de buena prescripción ${ }^{49}$, donde se analizan las diferentes fuentes de información y cómo elegirlas.

Actualmente hay plataformas sin ánimo de lucro de indudable interés nacidas con la finalidad de promover entre los profesionales sanitarios una información basada en la evidencia científica y no en la aportada por la industria farmacéutica; intentan que la relación entre las compañías farmacéuticas y los médicos cambie. La más famosa es la plataforma americana No free lunch ${ }^{50}$, con filiales en Reino Uni- do, Italia y actualmente en España (Plataforma No gracias $^{51}$.

Por último, mientras muchas organizaciones profesionales continúan dependiendo de la industria farmacéutica, hay otras que empiezan a desmarcarse de esta dependencia. Recientemente en Reino Unido, el Real Colegio de Médicos Generales intervino en una comisión en la Casa de los Comunes para la investigación y subsiguiente informe con recomendaciones para la industria farmacéutica². Por otro lado, se van creando documentos de cooperación entre la profesión médica y la industria farmacéutica que regulan la relación entre ambas partes de acuerdo a la legislación vigente ${ }^{52}$.

\section{Medios de comunicación}

Hay un creciente debate en los círculos periodísticos acerca de la propensión de los medios de comunicación a exagerar la prevalencia y gravedad de la enfermedad, siendo difícil en ocasiones distinguir entre la correcta y la incorrecta información. Frecuentemente, los efectos terapéuticos de los que se informan no son ni tan espectaculares ni tan desastrosos, y hay que ser conscientes de que los medios de comunicación son un importantísimo vehículo de promoción de nuevos avances terapéuticos y de nuevas enfermedades ${ }^{53}$. 
Debe ser un principio ético de los periodistas ofrecer una buena información a la población general. En este sentido, antes de lanzarse al sensacionalismo deberían asesorarse de profesionales bien formados, en relación a puntos clave ${ }^{6}$ :

- Definición correcta de la enfermedad, criterios diagnósticos apropiados y prevalencia real en la población general, en base a estudios científicos publicados.

- Conocimiento de los problemas y consecuencias del sobrediagnóstico. No se deberían utilizar anécdotas extremas, que no reflejan la enfermedad real, ni curas milagrosas, a pesar de que esto pueda "vender" menos.

- Cuantificación de los efectos secundarios de los fármacos.

- Actitud crítica con la esponsorización de la industria farmacéutica, que debe figurar siempre en la declaración de intereses.

Cada vez hay más fuentes de ayuda para que los revisores de medios de comunicación analicen si la información ofrecida en relación a una nueva enfermedad, una intervención o un fármaco, se adapta a criterios de calidad y cuál es la forma correcta de transmitirlo. El grupo Media Doctor (Canadá y Australia) y HealthNewsRevieW.org son organiza- ciones sin ánimo de lucro formadas por profesores universitarios, clínicos, editores y periodistas que revisan noticias médicas otorgando puntuaciones en base a criterios de correcta/incorrecta información, ayudando así a los consumidores a evaluar la evidencia, pros y contras de las noticias de salud ${ }^{54,55}$.

\section{Industria farmacéutica}

En un sentido positivo, hay algún cambio que parte desde la industria respecto a la forma de actuar con los profesionales y los pacientes. En 1991, Farmaindustria adoptó, como código español, el Código Europeo de Buenas Prácticas para la Promoción de Medicamentos. Tras varias adaptaciones posteriores, en 2005 se publica la versión definitiva de este código donde se regulan las formas y métodos de promoción de medicamentos a profesionales sanitarios que los prescriben y dispensan. No solo se comprometen acerca de cómo facilitar la información, con transparencia en la promoción, sino que regulan la incentivación a los profesionales, obsequios (siempre de valor insignificante), hospitalidad y reuniones científicas y entrega de muestras entre otros apartados. También contemplan un control y seguimiento de cumplimiento del código posterior ${ }^{56}$. 
Por otro lado han presentado, además, un nuevo código que establece cómo debe ser la relación con asociaciones de pacientes ${ }^{57}$. Es de esperar que esto no sea solo una declaración de intenciones como lavado de cara de la industria farmacéutica, sino una realidad.

\section{Políticos y Administración}

Poco a poco, las relaciones entre la industria y los médicos están teniendo su hueco en los debates de congresos de diversos países. Hace poco este tema ha sido objeto de análisis en el congreso estadounidense con críticas expresas por parte de senadores a Johnson Johnson y Eli Lilly Co, por los pagos a médicos de la Facultad de Medicina de Harvard que ayudaron a promover el uso de psicofármacos en jóvenes ${ }^{58}$.

Los políticos deben ser capaces de apoyar a una industria farmacéutica innovadora, que aporte indudables beneficios económicos y terapéuticos y proporcione empleo, pero deben estar en contra de aquella industria que trastorne y pueda llevar a la quiebra a un sistema universal de salud en su propio beneficio ${ }^{47}$. En este sentido es importante una seria regulación de anuncios de fármacos directos al público y de la relación de los profesionales sanitarios con las compañías farmacéuticas.
La Administración debe fundar y financiar organizaciones que provean información independiente y no sesgada en terapéutica. Paralelamente, debe arbitrar la manera de hacer llegar de forma eficiente esta información a los profesionales sanitarios y ofertar cobertura de las necesidades docentes que la profesión médica precisa para una correcta práctica clínica. De otra forma, y tal como ocurre actualmente, esta oferta se buscará en gran medida en otras organizaciones que pueden no ser tan independientes como sería deseable.

La provisión de información de alta calidad no sesgada es cara y forma parte de las Administraciones obtener la financiación adecuada para ello4.

\section{Pacientes y asociaciones}

Esta quizá sea la parte más vulnerable y más manipulable del problema; por eso la población y, sobre todo, las asociaciones de consumidores y pacientes, deben conocer la existencia de este fenómeno, entender los límites entre la normalidad y la enfermedad, y cuestionarse críticamente si son pacientes o víctimas. Es necesario que existan líderes responsables que se planteen cuánto hay de credibilidad en las historias y tratamientos médicos, sobre todo si se presentan a los pacientes como la cara hu- 
mana de una enfermedad que forma parte de la campaña de marketing arbitrada por los patrocinadores ${ }^{2}$.

\section{Epílogo}

No es banal, finalmente, que cada colectivo ponga su grano de arena. Los médicos, por un lado, deberíamos añadir una dosis de prudencia ante cualquier innovación, y por otro, independizar nuestra formación de la industria. Los gestores sanitarios deberían contemplar la formación de sus profesionales con la misma importancia que la asistencia, de forma que no sea casi tarea de héroes mantener una formación continuada, sin tener que acudir a la autofinanciación o a fuentes externas. Los políticos deben hacer una valoración ética y equitativa, y no solo económica de los ofrecimientos de la industria. $Y$ todos, como pacientes que antes o después seremos, debemos buscar la solución de los motivos de infelicidad, las imperfecciones de la mente y del cuerpo y los momentos de angustia, no solo ni fundamentalmente en la medicina. Sería prioritario indagar en la etiología psicosocial de muchos problemas como el TDAH en la infancia, volver al sentido común para tolerar las vicisitudes propias de cada etapa vital, como las inherentes a la adolescencia o la menopausia, y desde luego, buscar otra definición de salud que no nos enfrente a la frustración de buscar ese imposible y utópico estado de bienestar físico, psíquico y social que, según la OMS, es la salud.

\section{Bibliografía}

1. Fernández de Sanmamed MJ, Marquet R, Reguant M, Zapater F. ¿Enfermos de salud? Reflexiones acerca de las nuevas demandas y respuestas del sistema sanitario [monografía en Internet]. Barcelona: Grupo de Calidad de la CAMFYC; 2005 [consultado el 28/07/2008]. Disponible en www. camfic.org/grups_treball/docs/qualitat/enfer mos_salud_cast.pdf

2. Moynihan R, Henry D. The fight against disease mongering: generating knowledge for action. PLoS Med. 2006;3(4):e191.

3. Illich I. Limits to medicine: the exploration of health. New York: Penguin Books; 1977.
4. Shankar PR, Subish P. Disease mongering. Singapore Med J. 2007;48:275-80.

5. Payer L. Disease mongers: how doctors, drug companies and insurers are making you feel sick. New York: Wiley and Sons; 1992. p. 292.

6. Campos Requena V, Barrios Guerra C. Disease mongering [monografía en Internet]. Consumers Internacional; 2007 [consultado el 10/10/ 2008]. Disponible en www.consumersinternatio nal.org/files/98009/FileName/carlos_barrios_ 2007_03_15.pdf

7. Moynihan R, Heath I, Henry D. Selling sickness: the pharmaceutical industry and disease mongering. BMJ. 2002;324:886-91.

8. Wolinsky H. Disease mongering and drug 
marketing. EMBO Rep. 2005;6:612-4 [consultado el 23/03/2009]. Disponible en www.pubmedcen tral. nih.gov/articlerender. fcgi?artid=1369125

9. Collier J, Iheanacho I. The pharmaceutical industry as an informant. Lancet. 2002;360:1405-9.

10. Angell $M$. The truth about the drug companies: how they deceive us and what to do about it. New York: Random House; 2004.

11. Márquez Calderón S. La construcción y comunicación del conocimiento en la era de la Medicina Basada en la Evidencia. Implicaciones para la lectura crítica de ensayos clínicos. Ges Clin Sanit. 2006;8(5):87-93 [consultado el 23/03/2009]. Disponible en www.iiss.es/gcs/gestion29.pdf

12. Kaiser family Foundation (2005), Kaiser health poll report: public opinion snapshot on health information sources-July 2005. Menlo Park (California): Kaiser family foundation [consultado el 23/03/2009]. Disponible en www.kff.org/kaiser polls/pomr071805oth.cfm

13. Woloshin S, Schwartz LM. Giving legs to restless legs: a case study of how the media helps make people sick. PLoS Med. 2006;3(4):e170.

14. Nurnberg HG, Hensley PL, Heiman JR, Croft $H A$, Debattista C, Paine S. Sildenafil treatment of women with antidepressant-associated sexual dysfunction: a randomized controlled trial. JAMA. 2008;300:395-404.

15. Valerio M. Viagra también anima a las mujeres. El Mundo. Salud: martes 22 de julio de 2008 [consultado el 23/03/2009]. Disponible en www. elmundo.es/elmundosalud/2008/07/22/mu jer/1216748919.html

16. Elpais.com/efe. El principio activo de Viagra puede servir también a las mujeres. El País. Sociedad: domingo 27 de julio de 2008 [consultado el 03/03/2009].Disponible en www.elpais.com/arti culo/sociedad/principio/activo/Viagra/puede/ser vir/mujeres/elpepusoc/20080723elpepusoc_1/Tes

17. AFP Chicago. El Viagra eficaz para problemas sexuales de mujeres depresivas. ABC. Socie- dad: domingo 27 de julio de 2008 [consultado el 03/03/2009].Disponible en www.abc.es/hemero teca/historico-22-07-2008/abc/Sociedad/el-via gra-eficaz-para-problemas-sexuales-de-mujeresdepresivas-_1642019877162.html

18. Sanpedro J. Vendo enfermedades a la carta. Y remedios. El País. SoxciedadSociedad: jueves 9 de octubre de 2008 [consultado el 23/ 03/2009]. Disponible en www.elpais.com/articulo/socie dad/Vendo/enfermedades/carta/remedios/elpe pisoc/20081009elpepisoc_1/Tes?print=1

19. Caudill TS, Johnson MS, Rich EC, McKinney WP. Physicians, pharmaceutical sales representatives and the cost of prescribing. Arch Fam Med. 1996;5:201-6.

20. Moynihan R. Too much medicine? BMJ. 2002;324:859-60.

21. Abbasi K. No more free lunches. BMJ. 2003;326:1155-6.

22. Wikipedia [Internet]. Industria farmacéutica [consultado el 13/03/2009]. Disponible en http:// es.wikipedia.org/wiki/Industria_farmac\% C3\%A9 utica\#Cr.C3.ADticas_a_la_industria_farmac.C3.A9 utica

23. Márquez $S$, Meneu R. La medicalización de la vida y sus protagonistas. Ges Clin Sanit. 2003; 5(2):47-53 [consultado el 03/03/2009]. Disponible en www.iiss.es/gcs/gestion16.pdf

24. Baos Vicente V. La publicidad humanitaria: vacuna VPH, colchones. Blog El Supositorio [Internet]. Madrid [consultado el 03/03/2009]. Disponible en http://vicentebaos.blogspot.com/2008/08/ la-publicidad-humanitaria-vacuna-vph.html [actualizado el 29/08/2008].

25. Mintzes B. Disease mongering in drug promotion: do governments have a regulatory role? Disease mongering in drug promotion: do governments have a regulatory role? PLoS Med. 2006;3 (4):e198.

26. US Food and Drug Administration [Internet]. Silver Spring: Glaxo Wellcome decides to 
withdraw Lotronex form the market; 28 de noviembre de 2000 [consultado el 25/10/2008]. Disponible en www.fda.gov/bbs/topics/ANSWERS/ ANS01058.htlm

27. Stein DJ, Ipser JC, van Balkom AJ. Tratamiento farmacológico para la fobia social (Revisión Cochrane traducida). En: La Biblioteca Cochrane Plus, 2007 Número 4. Oxford: Update Software Ltd. Disponible en http://www.update-software. com. (Traducida de The Cochrane Library, 2007 Issue 4. Chichester, UK: John Wiley \& Sons, Ltd.).

28. Olivares J, Caballo V, García-López J, Alcázar R, López-Gollonet C. Una revisión de los estudios epidemiológicos sobre fobia social en población infantil adolescente y adulta. Psicol Conduct. 2003;11:405-27.

29. Mrakotsky C, Masek B, Biederman J, Raches $D, H$ sin $O$, Forbes $P$, et al. Prospective open-label pilot trial of mirtazapine in children and adolescents with social phobia. J Anxiety Disord. 2008:22:88-97.

30. Daniels SR, Greer FR, Committee on Nutrition. Lipid screening and cardiovascular health in childhood. Pediatrics. 2008;122;198-208.

31. De Ferranti S, Ludwig DS. Storm over statins. The controversy surrounding pharmacologic treatment of children. N Engl J Med. 2008;359: 1309-12.

32. Lexchin J. Bigger and better: how Pfizer redefined erectile dysfunction. PLoS Med. 2006;3 (4):e132.

33. Biederman J, Faraone SV. Attention-deficit hyperactivity disorder. Lancet. 2005;366:237-48.

34. Ruiz Canela J. Prevalencia del déficit de atención con hiperactividad (SAH). Pediatría Basada en la Evidencia [Internet]. 2003 [consultado el 04/03/2009]. Disponible en www.aepap.org/evi dencias/pdf/tvc_prevalencia_deficit_atencion.pdf

35. American Academy of Pediatrics. Comité on Quality Improvement, Subcommittee on Attention-Deficit/Hyperactivity Disorder Clinical Practice
Guideline. Diagnosis and evaluation of the child with attention-deficit/hyperactivity disorder. Pediatrics. 2000;105;1158-70.

36. Parens $E$, Johnston J. Mental health in children and adolescents. In: From birth to death and bench to clinic. The hastings center bioethics briefing book for journalists, policymakers, and campaigns. Mary Crowley ed. Garrison, NY: The Hastings Center; 2008. p. 101-06 [consultado el 04/ 03/2009]. Disponible en www.thehastingscen ter.org/uploadedFiles/Publications/Briefing_Bo ok/mental\%20heath\%20in\%20children \%20cha pter.pdf

37. Tizón García JL. El niño hiperactivo y su síndrome ¿mito, realidad, medicación? FMC. 2006; 13:1-4.

38. Center for Disease Control and Prevention. Mental Health in the United States: prevalence of diagnosis and treatment for attention-deficit/hyperactivity disorder. United States, 2003. MMWR. 2005;54:842-7.

39. Criado-Álvarez JJ, Romo-Barrientos C .Variabilidad y tendencias en el consumo de metilfenidato en España. Estimación de la prevalencia del trastorno por déficit de atención con hiperactividad. Rev Neurol. 2003;37:806-10.

40. Lasa Zulueta A. Hiperactividad y trastornos de la personalidad. Cuadernos de psiquiatría y psicoterapia del niño y del adolescente. 2001;3132:5-81.

41. Confias. Fundación para una infancia y adolescencia saludable [Internet] [consultado el 04/ 03/2009]. Disponible en www.fundacionconfias. org/

42. Ghodse H. Dramatic increase in methylphenidate consumption. Curr Opin Psytr. 1999;12:265-8.

43. Phillips CB. Medicine goes to school: teachersschool: teachers as sickness brokers for ADHD. PLoS Med. 2006;3(4):e182.

44. FDA talk paper [Internet]. Food and Drug Administration. Janssen Pharmaceutica stops mar- 
keting cisapride in the U.S. Department of Health and Human Services; March 23, 2000 [consultado el 02/03/2009]. Disponible en www.fda.gov/bbs/ topics/ANSWERS/ANS01007.html

45. EMEA. The European Agency for the Evaluation of Medicinal Products [Internet]. London, 2002. Committee for proprietary medicinal products (CPMP). Opinion following an article 31 referral Cisapride [consultado el 04/03/2009]. Disponible en www.emea.europa.eu/pdfs/human/ referral/cisapride/2484402en.pdf

46. Gervás J. Moderación en la actividad médica preventiva y curativa. Cuatro ejemplos de necesidad de prevención cuaternaria en España. Gac Sanit. 2006;20 (Supl 1):s127-s134.

47. Heath I. Combating disease mongering: daunting but nonetheless essential. PLoS Med. 2006:3(4):e146.

48. Gagnon MA, Lexchin J. The cost of pushing pills: a new estimate of pharmaceutical promotion expenditures in the United States. PLoS Med. 2008:5(1):e1.

49. De Vries TP, Henning RH, Hogerzeil HV, Fresle DA. Guide to good prescribing. WHO Action Programme on Essential Drugs [Internet]. Geneva: World Health Organization; 1994 [consultado el 04/12/2008]. Disponible en http://whqlib doc.who.int/hq/1994/WHO_DAP_94.11.pdf

50. No free lunch [Internet]. New York City: Goodman R [consultado el 10/10/2008]. Disponible en www.nofreelunch.org/

51. Nogracias.eu [Internet]. Federación de Asociaciones para la Defensa de la Sanidad Pública [consultado el 01/11/2008]. Disponible en www.nogracias.eu/v_portal/apartados/pl_basi ca.asp?te $=2309$

52. Declaración conjunta del CEPME y de la EFPIA sobre la cooperación entre la profesión médica y la industria farmacéutica [Internet]. Boletín Euro- pa al día. Consejo General de Colegios Oficiales de Médicos de España [consultado el 15/12/2008]. Disponible en www.cgcom.org/sites/default/files/ 167_cpme_efpia.pdf

53. Evaluating the media as a source of drug therapy information. Therapeutics Initiative [Internet]. Therapeutics letter 67-November-december 2007 [consultado el 15/12/2008]. Disponible en www.ti.ubc.ca/letter67

54. Health News Review.org. Grades for health news reporting [sede Web]. Schwitzer G. Foundation for informed medical decision making; 2008 [consultado el 20/12/2008]. Disponible en www. healthnewsreview.org/

55. Media Doctor Canada [Internet] [consultado el 22/12/2008]. Disponible en www.media doctor.ca/content/ratinginformation.jsp

56. Unidad de supervisión deontológica de la industria farmacéutica. Código español de buenas prácticas para la promoción de los medicamentos. Claves útiles para los profesionales sanitarios [Internet]. Madrid: Farmaindustria; 2005 [consultado el 15/12/2008]. Disponible en www.farmaindus tria.es/idc/groups/public/documents/c\%C3\%B3 digodocumento/farma_42142.pdf

57. Europa press Farmaindustria presenta un código de buenas prácticas que regulará sus relaciones con asociaciones de pacientes. Europapress.es. 23 de julio de 2008; Salud [consultado el 15/ 12/2008]. Disponible en www. europapress.es/salud/noticia-farmaindustriapresenta-codigo-buenas-practicas-regulara-re laciones-asociaciones-pacientes-2008072314 5408.html

58. Bloomberg Especial. Prohíben en EE. UU. los regalos que hacen los laboratorios a los médicos. Clarín Argentina. 11 de julio de 2008; Sociedad. Disponible en www.clarin.com/diario/2008/07/ 11/sociedad/s-01712662.htm 